\title{
Treatment of Capecitabine Corneal Side Effects With Autologous Blood-derived Serum Eye Drops
}

\author{
FEDERICO DI STASO ${ }^{1 *}$, IRENE GATTAZZO $^{1 *}$, BEATRICE TAURELLI SALIMBENI $^{2}$, \\ ALESSANDRO LAMBIASE ${ }^{3}$, GIANLUCA SCUDERI ${ }^{1}$, SILVIO DI STASO ${ }^{4}$ and MARCO CIANCAGLINI ${ }^{4}$ \\ ${ }^{1}$ Ophthalmology Unit, NESMOS Department, "Sapienza" University of Rome, Rome, Italy; \\ ${ }^{2}$ Department of Clinical and Molecular Medicine, University of Rome, Rome, Italy; \\ ${ }^{3}$ Department of Sense Organs, Policlinico Umberto I, Sapienza University of Rome, Rome, Italy; \\ ${ }^{4}$ Ophthalmic Clinic, Department of Life, Health and Environmental Sciences, University of L'Aquila, L'Aquila, Italy
}

\begin{abstract}
Background/Aim: To describe the clinical progress and management of ocular side effects in a 35-yearold patient with metastatic breast cancer who underwent oral chemotherapy with capecitabine and lapatinib. Materials and Methods: Slit lamp evaluation revealed bilateral perikeratic hyperemia, perilimbal conjunctival edema associated with corneal marginal infiltrates and epithelial and anterior stromal defects in both eyes. Slit lamp examination, in vivo confocal microscopy and anteriorsegment optical coherence tomography were highly suggestive for limbal stem cell deficiency. The decision to administer autologous blood-derived serum eye drops was made. Results: Following administration of autologous blood-derived serum eye drops, corneal marginal infiltrates, epithelial and stromal defects significantly regressed in both eyes after only 10 days. Chemotherapy was resumed and serum eye drops were prescribed simultaneously. Conclusion: Autologous blood-derived serum eye drops may be an adequate therapeutic choice for bilateral corneal lesions detected as ocular side effects of capecitabine.
\end{abstract}

Capecitabine (C), a fluoropyrimidine carbamate, is an antimetabolite agent indicated for the treatment of breast cancer refractory to paclitaxel or anthracycline. Xeloda (C, Hoffmann-La Roche, Basel, Switzerland) is administered

This article is freely accessible online.

*These Authors contributed equally to the present study.

Correspondence to: Federico Di Staso, Ophthalmology Unit, NESMOS Department, "Sapienza" University of Rome, Via di Boccea 190, 00167 Rome, Italy. Tel: +39 3920624768, e-mail: federico.distaso@gmail.com

Key Words: Capecitabine, chemotherapy, breast cancer, autologous serum, cornea, keratitis, confocal microscopy. orally as a prodrug and metabolized to fluorouracil (FU) in three steps. The enzyme responsible for the last step of drug activation is thymidine phosphorylase. This shows greater concentration in tumor cells where it produces pharmacological active levels of 5-FU, ultimately behaving as tumor-selective drug (1).

FDA has approved the use of capecitabine as first-line oral therapy for advanced and metastatic breast cancer either alone or in combination with other chemotherapeutic agents, most often taxanes. Due to its renal metabolism, $\mathrm{C}$ is contraindicated if creatinine clearance is $<30 \mathrm{ml} / \mathrm{min}$.

The most frequent adverse effects are hyperbilirubinemia (18\%), hand-foot syndrome

$(17 \%)$, diarrhea $(13 \%)$, ocular irritation (10\%), myelosuppression, fatigue and weakness, abdominal pain, and nausea; the most fatal side effect is cardiac toxicity, which has been reported in $1.2 \%$ to $18 \%$ of patients (2). Only $13 \%$ of patients presenting with such adverse events or intercurrent illnesses required treatment discontinuation (2).

Lapatinib (L) is a dual tyrosine kinase inhibitor that acts both on HER2 and EGFR. In 2007 and 2008 FDA and EMA, respectively, approved the use of $\mathrm{L}$ in in combination with $\mathrm{C}$ in patients with advanced HER2-positive breast cancer after failed therapy with anthracyclines, taxanes and trastuzumab. $\mathrm{L}$ is administered orally and generally well tolerated. The most frequently reported adverse effects are diarrhea, dyspepsia, and skin rash; more rarely hepatotoxicity and interstitial pneumonitis can occur, which require routine laboratory evaluation of liver function and clinical observation (3).

\section{Patients and Methods}

We present the case of a 34-year-old Caucasian woman with metastatic BC (HER2+, ER 70\%, PgR40\%, Ki67 50\%, ISH pos) who underwent bilateral mastectomy, followed by adjuvant chemotherapy with anti-HER2 agents and local radiotherapy to bone metastases. She did not have other past medical history and was not 
on any other regular medications. Due to disease progression her treatment was switched to $\mathrm{L}+\mathrm{C}$. This comprised a 21-day cycle of $\mathrm{L}$ at a dose of 1,250 mg per day and a 14-day course of $\mathrm{C}$ at cycle onset at a dose of $2,000 \mathrm{mg} / \mathrm{m}^{2}$ per day. This treatment was initially well tolerated with the exception of a mild form of hand-foot syndrome. After the 4 th cycle of $\mathrm{L}+\mathrm{C}$ the patient started to complain of blurry vision, foreign body sensation and eye redness not related to mechanical trauma.

She was therefore referred to our ophthalmology department, San Salvatore's Ophthalmic Unit in L'Aquila. Slit lamp evaluation revealed bilateral palpebral edema, conjunctival congestion, minimal discharge with perikeratic hyperemia associated with irregular and diffuse epithelial defects extending to the portion of anterior stroma in the superior corneal quadrants of both eyes.

Culture of secretions, polymerase chain reaction for Herpes Virus and screening for autoantibodies were negative.

Topical therapy with antibiotics and artificial tears was prescribed. Despite an initial mild improvement, eye conditions worsened immediately after the subsequent chemotherapy cycle. Bilateral perikeratic hyperemia, perilimbal conjunctival edema associated with corneal marginal infiltrates and epithelial and anterior stromal defects were detected, resembling a "whorl" pattern. In the superior half of the cornea fluorescein staining was strong and diffuse (Figure 1).

In vivo confocal microscopy (IVCM) examination was performed using the Rostock Cornea Module of the Heidelberg Retinal Tomograph III (Heidelberg Engineering GmbH, Heidelberg, Germany). The affected limbal and peri-limbal portions of the cornea showed an irregular cellular population and a mosaic pattern consisting mostly of hypo-reflective cells and cystic changes. The middle part of the lesion showed conjunctival-type epithelial cells mixed with subepithelial fibrosis (Figure 1). Anterior-segment optical coherence tomography (RTVue, Optovue Inc., Fremont, CA, USA) showed an irregular epithelial profile associated with hyperreflective signal on the same area (Figure 2).

Since clinical signs and imaging data were highly suggestive of limbal stem cell deficiency (LSCD), we decided to administer autologous blood-derived serum eye drops (SED).

The subsequent administration of chemotherapeutic agents was therefore delayed by one week in order to collect $50-100 \mathrm{ml}$ of peripheral blood with the lowest possible L+C serum concentrations. Blood was left for $2 \mathrm{~h}$ at room temperature to reach complete clotting. Serum was than separated by centrifugation at $3,000 \times g$ for $15 \mathrm{~min}$ under sterile conditions, filtered and stored without dilution at $-20^{\circ} \mathrm{C}$. Two drops of serum diluted with balanced saline solution at $30 \%$ were topically administered 5 times per day in both eyes.

\section{Results}

The patient was reassessed at 5 and 10 days. After 10 days her clinical conditions improved dramatically with significant regression of corneal marginal infiltrates, epithelial and stromal defects in both eyes. Fluorescein staining was limited to few small areas of residual epithelial defects (Figure 1). L+C therapy was resumed, and administration of topical autologous serum was continued simultaneously. After the $6^{\text {th }}$ cycle fluorescein staining and epithelial defects were absent. Only a small area of the anterior corneal stroma revealed a scar in the superior sector in both eyes.

$\mathrm{L}+\mathrm{C}$ therapy was maintained alongside SED without symptoms relapsing.

\section{Discussion}

The exact pathogenesis of $\mathrm{C}$ ocular toxicity is still not fully understood. C adverse effects are dose dependent and correlate with individual susceptibility and enzymatic deficiencies (4). Corneal toxicity had already been reported by Waikhom et al., where bilateral severe ocular irritation, and multiple white, granular subepithelial corneal deposits following two cycles of $\mathrm{C}$ had required treatment cessation due to severe visual impairment, with complete clearing of ocular signs within two months (5). C corneal toxicity has been demonstrated in dogs where it manifested as dosedependent superficial keratitis, characterized by multifocal geographic erosions, superficial epithelial pigmentation, and neovascularization. Ocular histopathologic analysis has revealed neovascularization and inflammatory infiltrate of the anterior corneal stroma and an alteration of basal cell morphology, along with disorganization, reduced thickness, and pigmentation of the corneal epithelium (6). Complete resolution of the corneal lesions has been observed following the interruption of the treatment with $\mathrm{C}$ in both dogs and human patients (5). Only one ocular adverse effect has been reported to be refractory to treatment cessation: a case of Cinduced bilateral cicatricial ectropion, which has required a surgical approach (7).

Interestingly, L seems to affect VEGF, one of the most important proteins in corneal neovascularization $(\mathrm{CN})$. In an experimental rat model, the anti-angiogenic effect of $\mathrm{L}$ has been demonstrated to significantly reduce corneal neovascular area, VEGF immunostaining intensities and corneal VEGF ELISA levels, thus preventing CN (8).

To our knowledge, this is the first time that SED have been used to treat $\mathrm{LSCD}$ related to $\mathrm{L}+\mathrm{C}$ and that related IVCM images have been provided. LSCD is due to depopulation and dysfunction of corneal epithelial limbal stem cells and is characterized by eye discomfort, recurrent epithelial defects, ocular surface inflammation, stromal ulceration, and scarring. Various causes have been considered responsible for this condition, such as chemical or thermal burns, chronic contact lens wear, surgery involving the limbus, infections, radiation and chemotherapy and toxic epidermal necrolysis (9). Each of these creates a sort of 'conjunctivalisation', which initially leads to the covering of the cornea by a layer of conjunctival cells accompanied by $\mathrm{CN}$ and later to the development of subepithelial fibrosis and a fibrovascular pannus with loss of vision in advanced stages. Recently, IVCM has gained a fundamental role in the discrimination between different 

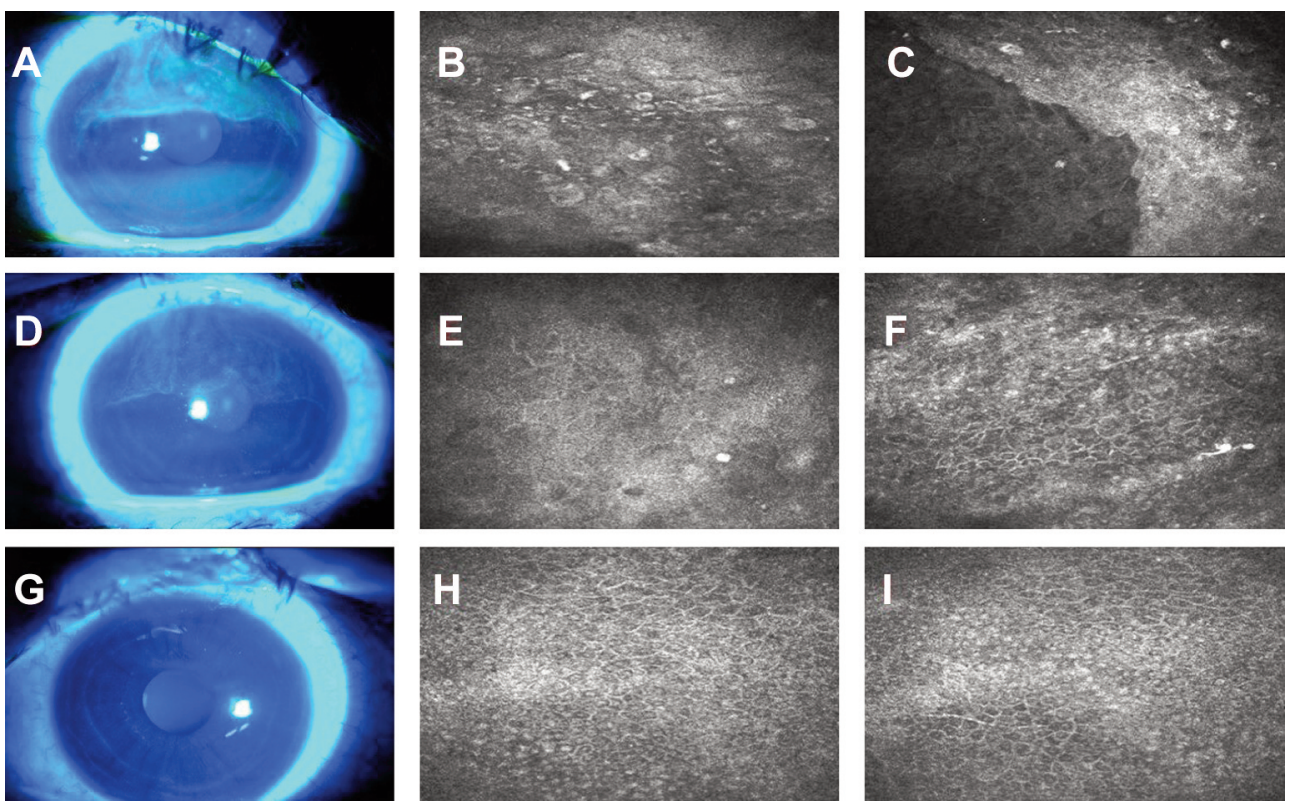

Figure 1. Clinical presentations and confocal images before and after treatment. A) Corneal epithelial defect shown by fluorescein dye and cobalt blue light resembling a "curtain" pattern. B) The limbal side of the epithelial layer appears with irregular cellular architecture and mosaic pattern consisting of hypo-reflective cells along with cystic changes. C) In vivo confocal microscopy at the edge of the damaged cornea shows bright conjunctival-type epithelial cells mixed with subepithelial fibrosis opposite to darker corneal type epithelial cells. D) Attenuation of the corneal epithelial defect demonstrated by a less intense fluorescein dye staining after 5 days. E, F) Healing corneal epithelium composed by polygonal cells with hypo-reflective cytoplasm and barely detectable nuclei. Hyper-reflective cells may be interpreted as residual goblet cells. G) Only a small residual epithelial defect is visible in the superior portion of the affected cornea after 10 days. $\mathrm{H}, \mathrm{I})$ corneal-type epithelium with polygonal well defined cell borders and brighter nuclei.

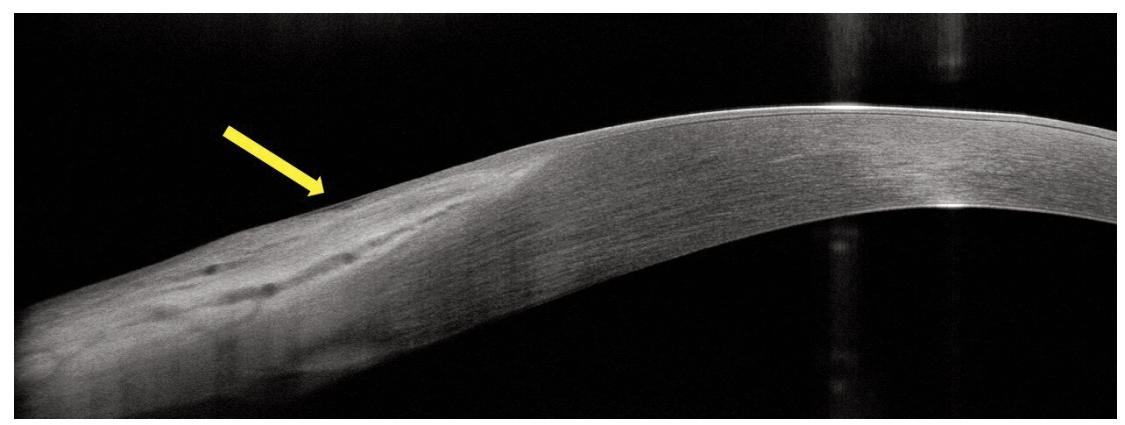

Figure 2. Anterior segment-optical coherence tomography image of the affected area. Yellow arrow: irregular epithelial profile with mild subepithelial fibrosis.

types of epithelium, cell phenotypes and the detection of the corneal-conjunctival epithelial transition, appearing more reliable than biomicroscopy and impression cytology (10).

Due to the patient's clinical status, we decided not to interrupt L+C chemotherapy, but to contrast its ocular adverse effects using autologous SED. The high concentration of epitheliotrophic growth factors released by the degranulation of platelet alpha-granules during the clotting of proteins, carbohydrates, lipids, and various electrolytes, makes blood-derived serum eye drops very similar to tear composition. Thus, they have been shown to maintain and restore ocular surface integrity (11). A similar situation was observed in another patient with breast cancer on trastuzumab, where ocular side effects, such as limbal lesions and corneal erosion, were successfully treated by SED (12). 
The exact mechanism of corneal damage and erosion is not known but the serum strategy employed in this case allowed the patient to continue the anti-cancer treatment. Even if the pathogenesis of corneal damage is not completely understood, we hypothesize that chemotherapy may have somehow impaired the normal epithelial turnover and that the autologous serum may have enhanced epithelial regrowth.

A single case report does not clearly demonstrate the mechanism of action of patient-derived serum, but the successful clinical result warrants further studies given the number of patients undergoing chemotherapy every day.

\section{Conflicts of Interest}

The Authors do not have any conflicts of interest to declare in relation to this study.

\section{Authors' Contributions}

Conceptualization, I.G. and F.D.S.; manuscript writing I.G. and F.D.S.; resources, B.T.S.; validation, A.L., supervision, G.S., S.D.S. and M.C.

\section{Acknowledgements}

The Authors thank Matthew Hastings and Nives Gattazzo for proofreading the manuscript.

\section{References}

1 Blum JL: Xeloda in the treatment of metastatic breast cancer. Oncology 57(Suppl 1): 16-20, 1999. PMID: 10436412. DOI: $10.1159 / 000055264$

2 Walko CM and Lindley C: Capecitabine: A review. Clin Ther 27(1): 23-44, 2005. PMID: 15763604. DOI: 10.1016/j.clinthera. 2005.01.005

3 Voigtlaender M, Schneider-Merck T and Trepel M: Lapatinib. Recent Results Cancer Res 211: 19-44, 2018. PMID: 30069757. DOI: 10.1007/978-3-319-91442-8_2

4 Reigner B, Blesch K and Weidekamm E: Clinical pharmacokinetics of capecitabine. Clin Pharmacokinet 40(2): 85-104, 2001. PMID: 11286326. DOI: 10.2165/00003088-200140020-00002
5 Waikhom B, Fraunfelder FT and Henner WD: Severe ocular irritation and corneal deposits associated with capecitabine use. N Engl J Med 343(10): 740-741, 2000. PMID: 10979776. DOI: 10.1056/NEJM200009073431015

6 Zarfoss M, Bentley E, Milovancev M, Schmiedt C, Dubielzig R and McAnulty J: Histopathologic evidence of capecitabine corneal toxicity in dogs. Vet Pathol 44(5): 700-702, 2007. PMID: 17846245. DOI: $10.1354 / \mathrm{vp} .44-5-700$

7 Tatar S, Yalçın CE, Sezgin B, Taş AY, Müftüoğlu O and Özmen S: Capecitabine-induced bilateral ectropion: A rare ocular manifestation requiring surgical intervention. J Cutan Aesthet Surg 11(4): 241-244, 2018. PMID: 30886481. DOI: 10.4103/JCAS.JCAS_5_18

8 Kaya MK, Demir T, Bulut H, Akpolat N and Turgut B: Effects of lapatinib and trastuzumab on vascular endothelial growth factor in experimental corneal neovascularization. Clin Exp Ophthalmol 43(5): 449-457, 2015. PMID: 25640924. DOI: 10.1111/ceo.12500

9 Deng SX, Borderie V, Chan CC, Dana R, Figueiredo FC, Gomes JAP, Pellegrini G, Shimmura S, Kruse FE and and the International Limbal Stem Cell Deficiency Working Group: Global consensus on definition, classification, diagnosis, and staging of limbal stem cell deficiency. Cornea 38(3): 364-375, 2019. PMID: 30614902. DOI: 10.1097/ICO.0000000000001820

10 Nubile M, Lanzini M, Miri A, Pocobelli A, Calienno R, Curcio C, Mastropasqua R, Dua HS and Mastropasqua L: In vivo confocal microscopy in diagnosis of limbal stem cell deficiency. Am J Ophthalmol 155(2): 220-232, 2013. PMID: 23127748. DOI: 10.1016/j.ajo.2012.08.017

11 Bernabei F, Roda M, Buzzi M, Pellegrini M, Giannaccare G and Versura P: Blood-based treatments for severe dry eye disease: The need of a consensus. J Clin Med 8(9):1478, 2019. PMID: 31533289. DOI: $10.3390 / \mathrm{jcm} 8091478$

12 Orlandi A, Fasciani R, Cassano A, Agresta A, Calegari MA, Caporossi A and Barone C: Trastuzumab-induced corneal ulceration: Successful no-drug treatment of a "blind" side effect in a case report. BMC Cancer 15: 973, 2015. PMID: 26672594. DOI: $10.1186 / \mathrm{s} 12885-015-1969-3$ 